\title{
A fixed point theorem for systems of operator equations and its application
}

\author{
Yujun Cui \\ State Key Laboratory of Mining Disaster Prevention and Control Co-founded by Shandong Province and the Ministry of Science and \\ Technology, Shandong University of Science and Technology, Qingdao 266590, P. R. China.
}

Communicated by N. Hussain

\begin{abstract}
A new fixed point theorem in product cones is established for systems of operator equations, where the components are expressed by partial ordering. In applications, this allows the nonlinear term of a differential system to have different behaviors in components. (C)2017 All rights reserved.
\end{abstract}

Keywords: Fixed point theorem, differential system, partial order. 2010 MSC: $47 \mathrm{H} 10$.

\section{Introduction}

Let $E$ be a Banach space, $P \subset E$ be a cone. Then $E$ becomes an ordered Banach space equipped with the partial ordering " $\leqslant$ " induced by P. P is called normal if there exists a constant $N>0$ such that $\theta \leqslant x \leqslant y$ implies $\|x\| \leqslant N\|y\|$. One can refer the concepts and the properties of the cone to [8, 10]. In this paper we investigate the existence of fixed point for systems of the operator equations

$$
\left\{\begin{array}{l}
u_{1}=N_{1}\left(u_{1}, u_{2}\right) \\
u_{2}=N_{2}\left(u_{1}, u_{2}\right)
\end{array}\right.
$$

where $N_{i} \in C[E \times E, E](i=1,2)$.

Recently, much work has been carried out on the existence of positive solutions of various type of nonlinear problems (see $[1-7,9,11,15-25])$. One of the most common approaches is to set down an equivalent abstract operator for nonlinear problems and then applying the topological degree, the fixed point theorem or the fixed point index theory in cones to get the desired results. For example, in [16, 23], the authors applied the fixed point index theory to study the existence and multiplicity of positive solutions of boundary value problems for systems of second order ordinary differential equations. Precup in [18] considered the existence of positive periodic solutions of a first order differential system by establishing a new version of Krasnosel'ski1̌'s fixed point theorem in cones for systems of operator equations, where the compression-expansion conditions are expressed on components. In [4], the authors discussed the

Email address: cyj720201@163.com (Yujun Cui)

doi:10.22436/jnsa.010.11.31 
multiplicity of positive solutions for a class of $\left(p_{1}, p_{2}\right)$-Laplacian systems based on the product formula of the fixed point index and Leray-Schauder degree theory.

Motivated by works mentioned above, we employ the e-positive operator or/and functional and the partial ordering to get some new fixed point theorem for operator equation (1.1). As application of our main result, the existence of positive solutions for system of second and fourth order ordinary differential equations is considered.

\section{Main results}

We first recall some concepts and conclusions on the fixed point index in $[8,10]$ for the proof of fixed point theorem. Let $E$ be a Banach space and let $P \subset E$ be a cone in $E$. Assume that $\Omega$ is a bounded open subset of $E$ with boundary $\partial \Omega$, and let $A: P \cap \Omega \rightarrow P$ be a completely continuous operator. If $A u \neq u$ for $u \in P \cap \partial \Omega$, then the fixed point index $i(A, P \cap \Omega, P)$ can be defined. One important fact is that if $i(A, P \cap \Omega, P) \neq 0$, then $A$ has a fixed point in $P \cap \Omega$.

The following lemmas is needed in our proofs.

Lemma $2.1([8,10])$. Let $\mathrm{A}: \mathrm{P} \rightarrow \mathrm{P}$ be completely continuous. We have

(i) if $\mathrm{A} u \neq \mu \mathrm{u}$ for all $\mathrm{u} \in \mathrm{P} \cap \partial \Omega$ and $\mu \geqslant 1$, then $\mathrm{i}(\mathrm{A}, \mathrm{P} \cap \Omega, \mathrm{P})=1$;

(ii) if there exists $\mathrm{u}_{0} \in \mathrm{P}, \mathrm{u}_{0} \neq \theta$ such that $\mathrm{u}-\mathrm{A} u \neq \mu \mathrm{u}_{0}$ for all $\mathrm{u} \in \mathrm{P} \cap \partial \Omega$ and $\mu \geqslant 0$, then $\mathrm{i}(\mathrm{A}, \mathrm{P} \cap \Omega, \mathrm{P})=0$.

Lemma 2.2 ([4]). Let $\mathrm{E}$ be a real Banach space, $\mathrm{P}_{i} \subset \mathrm{E}$ be a cone and $\mathrm{W}_{i}$ be a bounded open subset of $\mathrm{E}$ with boundary $\partial W_{i}(i=1,2)$. Suppose that $A_{i}: P_{i} \cap W_{i} \rightarrow P_{i}$ is a completely continuous operator and that $A_{i} u_{i} \neq u_{i}$, for all $\mathrm{u}_{\mathrm{i}} \in \mathrm{P}_{\mathrm{i}} \cap \partial \mathrm{W}_{\mathrm{i}}$. Then

$$
i\left(A,\left(P_{1} \times P_{2}\right) \cap\left(W_{1} \times W_{2}\right), P_{1} \times P_{2}\right)=i\left(A_{1}, P_{1} \cap W_{1}, P_{1}\right) \cdot i\left(A_{2}, P_{2} \cap W_{2}, P_{2}\right),
$$

where $A(u, v)=\left(A_{1} u, A_{2} v\right), \forall(u, v) \in\left(P_{1} \times P_{2}\right) \cap\left(W_{1} \times W_{2}\right)$.

In what follows, we shall consider two normal cones $P_{1}, P_{2}$ of a Banach space $E$, normal cone $K_{1}$ of a Banach space $\left(E_{1},\|\cdot\|_{1}\right)$ with normal constant $\bar{N}_{1}$, normal cone $K_{2}$ of a Banach space $\left(E_{2},\|\cdot\|_{2}\right)$ with normal constant $\bar{N}_{2}$, cone $P_{1} \times P_{2}$ of a Banach space $E \times E$ with the norm $\left\|\left(u_{1}, u_{2}\right)\right\|=\max \left\{\left\|u_{1}\right\|,\left\|u_{2}\right\|\right\}$, and we shall use the same symbol " $\leqslant$ " to denote the partial ordering relations induced by $\mathrm{P}_{1} \times \mathrm{P}_{2}$ in $E \times E$, and by $P_{1}, P_{2}, K_{1}, K_{2}$ in $E, E_{1}$ and $E_{2}$.

Definition 2.3. Let $P$ be a cone in a Banach space $E$, and $K_{1}$ be a cone in a Banach space $E_{1}$. Let $e \in K_{1} \backslash\{\theta\}$. A linear operator $\mathrm{T}: \mathrm{P} \rightarrow \mathrm{K}_{1}$ is called e-positive, if for every nonzero $\mathrm{x} \in \mathrm{P}$ there exist two positive number $c(x), d(x)$ such that

$$
c(x) e \leqslant T x \leqslant d(x) e .
$$

Remark 2.4. The above definition of e-positive operator is different from the previous definition in $[12,13]$. The main difference is that $E \neq E_{1}$, or $E=E_{1}$ but $P \neq K_{1}$. When $E=E_{1}$ and $P \subset K_{1}$, Definition 2.3 is the same as [21, Definition 2.2].

We now state and prove the main abstract result of this paper.

Theorem 2.5. Let $\mathrm{N}=\left(\mathrm{N}_{1}, \mathrm{~N}_{2}\right): \mathrm{P}_{1} \times \mathrm{P}_{2} \rightarrow \mathrm{P}_{1} \times \mathrm{P}_{2}$ be a completely continuous operator. Assume that for each $i \in\{1,2\}$ one of the following conditions is satisfied:

(a) There exist $k_{i 1} \in(1,+\infty), k_{i 2} \in[0,1), k_{i}, r_{i}, M_{i} \in(0,+\infty), e_{i} \in K_{i} \backslash\{\theta\}$ and two linear $e_{i}$-positive operators $\mathrm{B}_{\mathrm{i} 1}, \mathrm{~B}_{\mathrm{i} 2}: \mathrm{P}_{\mathrm{i}} \rightarrow \mathrm{K}_{\mathrm{i}}$, such that

$$
B_{i 1} N_{i}(u) \geqslant k_{i 1} B_{i 1} u_{i}, \quad\left\|u_{i}\right\| \leqslant r_{i}, \quad u=\left(u_{1}, u_{2}\right) \in P_{1} \times P_{2}, \quad B_{i 2} N_{i}(u) \leqslant k_{i 2} B_{i 2} u_{i}+M_{i} e_{i},
$$

and

$$
\left\|B_{i 2} u_{i}\right\|_{i} \geqslant k_{i}\left\|u_{i}\right\|, \quad u_{i} \in P_{i} .
$$

(b) There exist $k_{i 1} \in[0,1), k_{i 2} \in(1,+\infty), k_{i}, r_{i}, M_{i} \in(0,+\infty), e_{i} \in K_{i} \backslash\{\theta\}$ and two $e_{i}$-positive operators 
$\mathrm{B}_{\mathrm{i} 1}, \mathrm{~B}_{\mathrm{i} 2}: \mathrm{P}_{\mathrm{i}} \rightarrow \mathrm{K}_{\mathrm{i}}$ such that

$$
B_{i 1} N_{i}(u) \leqslant k_{i 1} B_{i 1} u_{i}, \quad\left\|u_{i}\right\| \leqslant r_{i}, \quad u=\left(u_{1}, u_{2}\right) \in P_{1} \times P_{2}, \quad B_{i 2} N_{i}(u) \geqslant k_{i 2} B_{i 2} u_{i}-M_{i} e_{i},
$$

and

$$
\left\|B_{i 2} u_{i}\right\|_{i} \geqslant k_{i}\left\|u_{i}\right\|, \quad u_{i} \in P_{i} .
$$

Then $\mathrm{N}$ has a fixed point $\mathrm{u}$ in $\mathrm{P}_{1} \times \mathrm{P}_{2}$ with $\left\|u_{i}\right\|>0(i=1,2)$.

Proof. We prove Theorem 2.5 for the following case:

$$
\begin{aligned}
& B_{11} N_{1}(u) \geqslant k_{11} B_{11} u_{1}, \quad\left\|u_{1}\right\| \leqslant r_{1}, \\
& B_{12} N_{1}(u) \leqslant k_{12} B_{12} u_{1}+M_{1} e_{1}, \quad u \in P_{1} \times P_{2}, \\
& B_{21} N_{2}(u) \leqslant k_{21} B_{21} u_{2}, \quad\left\|u_{2}\right\| \leqslant r_{2}, \\
& B_{22} N_{2}(u) \geqslant k_{22} B_{22} u_{2}-M_{2} e_{2}, \quad u \in P_{1} \times P_{2} .
\end{aligned}
$$

Since the proof of other cases are analogous to the above case, we omit it.

Let

$$
\begin{aligned}
R_{1} & =\max \left\{\frac{M_{1} \bar{N}_{1}\left\|e_{1}\right\|_{1}}{k_{1}\left(1-k_{12}\right)}+1, r_{1}+1\right\}, \quad R_{2}=\max \left\{\frac{M_{2} \bar{N}_{2}\left\|e_{2}\right\|_{2}}{k_{2}\left(k_{22}-1\right)}+1, r_{2}+1\right\}, \\
U & =\left(B_{R_{1}} \backslash \overline{B_{\frac{r_{1}}{2}}}\right) \times\left(B_{R_{2}} \backslash \overline{B_{\frac{r_{2}}{2}}}\right)=\left\{\left(u_{1}, u_{2}\right) \in P_{1} \times P_{2}: \frac{r_{i}}{2}<\left\|u_{i}\right\|<R_{i}, \quad i=1,2\right\} .
\end{aligned}
$$

Clearly, $U$ is an open set of $P_{1} \times P_{2}$. For $t \in[0,1]$, we consider the completely continuous homotopy $\mathrm{H}:[0,1] \times \overline{\mathrm{U}} \rightarrow \mathrm{P}_{1} \times \mathrm{P}_{2}$ defined by

$$
H(t, u)=\left(N_{1}\left(u_{1}, t u_{2}\right), N_{2}\left(t u_{1}, u_{2}\right)\right), \quad u=\left(u_{1}, u_{2}\right) \in P_{1} \times P_{2} .
$$

We will prove that $\{H(t, u)\}_{t \in[0,1]}$ satisfy $H(t, u) \neq u$ for each $t \in[0,1]$ and $u \in \partial u=\left\{\left(u_{1}, u_{2}\right) \in\right.$ $\mathrm{P}_{1} \times \mathrm{P}_{2}:\left\|\mathrm{u}_{1}\right\|=\frac{\mathrm{r}_{1}}{2}$ or $\left\|\mathrm{u}_{1}\right\|=\mathrm{R}_{1}$ or $\left\|\mathrm{u}_{2}\right\|=\frac{\mathrm{r}_{2}}{2}$ or $\left.\left\|\mathrm{u}_{2}\right\|=\mathrm{R}_{2}\right\}$, the sufficient conditions for the homotopy invariance of fixed point index on $\partial \mathrm{U}$. Next, we separate the proof of Theorem 2.5 into four steps.

Step 1. We claim that

$$
u_{1}-N_{1}\left(u_{1}, t u_{2}\right) \neq \mu \widetilde{e_{1}}, \quad \mu \geqslant 0, \quad t \in[0,1] \text { and }\left(u_{1}, u_{2}\right) \in \partial B_{\frac{r_{1}}{2}} \times P_{2},
$$

where $\widetilde{e_{1}}$ is a fixed element in $P_{1} \backslash\{\theta\}$. In fact, if there exist $\mu \geqslant 0, t \in[0,1]$ and $\left(u_{1}, u_{2}\right) \in \partial B_{\frac{r_{1}}{2}} \times P_{2}$ such that $u_{1}-N_{1}\left(u_{1}, t u_{2}\right)=\mu \widetilde{e_{1}}$, then from (2.1), one deduces that

$$
B_{11} u_{1}=B_{11} N_{1}\left(u_{1}, t u_{2}\right)+\mu B_{11} \widetilde{e_{1}} \geqslant B_{11} N_{1}\left(u_{1}, t u_{2}\right) \geqslant k_{11} B_{11} u_{1},
$$

that is, $\left(k_{11}-1\right) B_{11} u_{1} \leqslant \theta$. Notice that $k_{11}>1$, by the definition of $e_{i}$-positive operator, we obtain that $\mathrm{u}_{1}=\theta$, which is a contradiction with $u \in \partial \mathrm{B}_{\frac{r_{1}}{2}}$.

Step 2. We claim that

$$
\mathrm{N}_{1}\left(\mathrm{u}_{1}, \mathrm{t} \mathrm{u}_{2}\right) \neq \mu \mathrm{u}_{1}, \quad \mu \geqslant 1, \quad \mathrm{t} \in[0,1] \text { and }\left(\mathrm{u}_{1}, \mathrm{u}_{2}\right) \in \partial \mathrm{B}_{\mathrm{R}_{1}} \times \mathrm{P}_{2} .
$$

Suppose that there exist $\mu \geqslant 1, t \in[0,1]$ and $\left(u_{1}, u_{2}\right) \in \partial B_{R_{1}} \times P_{2}$ with $\mu u_{1}=N_{1}\left(u_{1}, t u_{2}\right)$. So, by (2.2), we have

$$
B_{12} u_{1} \leqslant \mu B_{12} u_{1}=B_{12} N_{1}\left(u_{1}, t u_{2}\right) \leqslant k_{12} B_{12} u_{1}+M_{1} e_{1}
$$

As a result,

$$
\left(1-k_{12}\right) B_{12} u_{1} \leqslant M_{1} e_{1}
$$

Considering the normality of cone, we get

$$
k_{1}\left(1-k_{12}\right)\left\|u_{1}\right\| \leqslant\left\|\left(1-k_{12}\right) B_{12} u_{1}\right\|_{1} \leqslant \bar{N}_{1}\left\|M_{1} e_{1}\right\|_{1},
$$

which implies that $\left\|u_{1}\right\| \leqslant \frac{\bar{N}_{1} M_{1}\left\|e_{1}\right\|_{1}}{k_{1}\left(1-k_{12}\right)}<R_{1}$. This contradicts $u_{1} \in \partial B_{R_{1}}$. 
Step 3. We claim that

$$
\mu u_{2} \neq N_{2}\left(t u_{1}, u_{2}\right), \quad \mu \geqslant 1, \quad t \in[0,1] \text { and }\left(u_{1}, u_{2}\right) \in P_{1} \times \partial B_{\frac{r_{2}}{2}} .
$$

If this is false, then there exist $\mu \geqslant 1, t \in[0,1]$ and $\left(u_{1}, u_{2}\right) \in P_{1} \times \partial B_{\frac{r_{2}}{2}}$ such that $\mu u_{2}=N_{2}\left(t u_{1}, u_{2}\right)$. This together with (2.3) yields

$$
B_{21} u_{2} \leqslant \mu B_{21} u_{2}=B_{21} N_{2}\left(t u_{1}, u_{2}\right) \leqslant k_{21} B_{21} u_{2}<B_{21} u_{2},
$$

a contradiction follows from the definition of $e_{i}$-positive operator and $u_{2} \in P_{2} \backslash\{\theta\}$. So, (2.7) holds.

Step 4. We claim that

$$
u_{2}-N_{2}\left(t u_{1}, u_{2}\right) \neq \mu \widetilde{e_{2}}, \quad \mu \geqslant 0, \quad t \in[0,1] \text { and }\left(u_{1}, u_{2}\right) \in P_{1} \times \partial B_{R_{2}},
$$

where $\widetilde{e_{2}}$ is a fixed element in $P_{2} \backslash\{\theta\}$. Suppose that there exist $\mu \geqslant 0, t \in[0,1]$ and $\left(u_{1}, u_{2}\right) \in P_{1} \times \partial B_{R_{2}}$ with $u_{2}-N_{2}\left(t u_{1}, u_{2}\right)=\mu \widetilde{e_{2}}$. Therefore by (2.4), we have

$$
B_{22} u_{2}=B_{22} N_{2}\left(t u_{1}, u_{2}\right)+\mu B_{22} \widetilde{e_{2}} \geqslant B_{22} N_{2}\left(t u_{1}, u_{2}\right) \geqslant k_{22} B_{22} u_{2}-M_{2} e_{2},
$$

which implies

$$
M_{2} e_{2} \geqslant\left(k_{22}-1\right) B_{22} u_{2}
$$

In view of the normality of cone, we get

$$
\bar{N}_{2} M_{2}\left\|e_{2}\right\|_{2} \geqslant\left(k_{22}-1\right)\left\|B_{22} u_{2}\right\|_{2} \geqslant k_{2}\left(k_{22}-1\right)\left\|u_{2}\right\| .
$$

This guarantees

$$
\left\|u_{2}\right\| \leqslant \frac{\bar{N}_{2} M_{2}\left\|e_{2}\right\|_{2}}{k_{2}\left(k_{22}-1\right)}<R_{2}
$$

which is a contradiction. Hence (2.8) is true.

Based on the expressions (2.5), (2.6), (2.7) and (2.8), it is not difficult to verify that $\{H(t, u)\}_{t \in[0,1]}$ satisfy the sufficient conditions for the homotopy invariance of fixed point index on $\partial \mathrm{U}$. Moreover, by Lemma 2.1, we have

$$
\begin{aligned}
& i\left(N_{1}(\cdot, \theta), B_{R_{1}}, P_{1}\right)=1, \quad i\left(N_{1}(\cdot, \theta), B_{\frac{r_{1}}{2}}, P_{1}\right)=0, \\
& i\left(N_{2}(\theta, \cdot), B_{R_{2}}, P_{2}\right)=0, \quad i\left(N_{2}(\theta, \cdot), B_{\frac{r_{2}}{2}}, P_{2}\right)=1 .
\end{aligned}
$$

Then from the homotopy invariance property of fixed point index and Lemma 2.2, we have

$$
\begin{aligned}
i\left(H(1, \cdot),\left(B_{R_{1}} \backslash \overline{B_{\frac{r_{1}}{2}}}\right)\right. & \left.\times\left(B_{R_{2}} \backslash \overline{B_{\frac{r_{2}}{2}}}\right), P_{1} \times P_{2}\right) \\
& =i\left(H(0, \cdot),\left(B_{R_{1}} \backslash \overline{B_{\frac{r_{1}}{2}}}\right) \times\left(B_{R_{2}} \backslash \overline{B_{\frac{r_{2}}{2}}}\right), P_{1} \times P_{2}\right) \\
& =i\left(N_{1}(\cdot, \theta), B_{R_{1}} \backslash \overline{B_{\frac{r_{1}}{2}}}, P_{1}\right) \cdot i\left(N_{2}(\theta, \cdot), B_{R_{2}} \backslash \overline{B_{\frac{r_{2}}{2}}}, P_{2}\right) \\
& =\left(i\left(N_{1}(\cdot, \theta), B_{R_{1}}, P_{1}\right)-i\left(N_{1}(\cdot, \theta), B_{\frac{r_{1}}{2}}, P_{1}\right) \cdot\left(i\left(N_{2}(\theta, \cdot), B_{R_{2}}, P_{2}\right)-i\left(N_{2}(\theta, \cdot), B_{\frac{r_{2}}{2}}, P_{2}\right)\right)\right. \\
& =-1 .
\end{aligned}
$$

Thus we are able to apply the solution property of the fixed point index to show that $\mathrm{N}$ has a fixed point $u$ in $P_{1} \times P_{2}$ with $\left\|u_{i}\right\|>0(i=1,2)$.

Remark 2.6. In Theorem 2.5 other three cases are possible for $u=\left(u_{1}, u_{2}\right) \in P_{1} \times P_{2}$ : 
(C1) $B_{11} N_{1}(u) \geqslant k_{11} B_{11} u_{1},\left\|u_{1}\right\| \leqslant r_{1} ; B_{12} N_{1}(u) \leqslant k_{12} B_{12} u_{1}+M_{1} e_{1}, u \in P_{1} \times P_{2}$, $B_{21} N_{2}(u) \geqslant k_{21} B_{21} u_{2},\left\|u_{2}\right\| \leqslant r_{2} ; B_{22} N_{2}(u) \leqslant k_{22} B_{22} u_{2}+M_{2} e_{2}, u \in P_{1} \times P_{2}$.

(C2) $B_{11} N_{1}(u) \leqslant k_{11} B_{11} u_{1},\left\|u_{1}\right\| \leqslant r_{1} ; B_{12} N_{1}(u) \geqslant k_{12} B_{12} u_{1}-M_{1} e_{1}, u \in P_{1} \times P_{2}$, $B_{21} N_{2}(u) \geqslant k_{21} B_{21} u_{2},\left\|u_{2}\right\| \leqslant r_{2} ; B_{22} N_{2}(u) \leqslant k_{22} B_{22} u_{2}+M_{2} e_{2}, u \in P_{1} \times P_{2}$.

(C3) $B_{11} N_{1}(u) \leqslant k_{11} B_{11} u_{1},\left\|u_{1}\right\| \leqslant r_{1} ; B_{12} N_{1}(u) \geqslant k_{12} B_{12} u_{1}-M_{1} e_{1}, u \in P_{1} \times P_{2}$, $B_{21} N_{2}(u) \leqslant k_{21} B_{21} u_{2},\left\|u_{2}\right\| \leqslant r_{2} ; B_{22} N_{2}(u) \geqslant k_{22} B_{22} u_{2}-M_{2} e_{2}, u \in P_{1} \times P_{2}$.

Theorem 2.7. The conclusion of Theorem 2.5 also holds if condition (b) is replaced with

$\left(b^{\prime}\right)$ There exist $k_{i 1} \in[0,1), k_{i 2} \in(1,+\infty), r_{1}, r_{2} \in(0,+\infty)$ with $r_{1}<r_{2}, e_{i} \in K_{i}$ and two $e_{i}$-positive operators $\mathrm{B}_{\mathrm{i} 1}, \mathrm{~B}_{\mathrm{i} 2}$ such that

$$
B_{i 1} N_{i}(u) \leqslant k_{i 1} B_{i 1} u_{i}, \quad\left\|u_{i}\right\| \leqslant r_{1},
$$

and

$$
B_{i 2} N_{i}(u) \geqslant k_{i 2} B_{i 2} u_{i}, \quad\left\|u_{i}\right\| \geqslant r_{2}
$$

\section{Application}

In this section, we shall apply Theorem 2.5 to the existence of positive solution for system of second and fourth order ordinary differential equations

$$
\left\{\begin{array}{l}
u_{1}^{(4)}=g_{1}(t) f_{1}\left(t, u_{1}, u_{2}\right), \quad t \in I=[0,1] \\
u_{2}^{\prime \prime}=-g_{2}(t) f_{2}\left(t, u_{1}, u_{2}\right), \\
u_{1}(0)=u_{1}(1)=u_{1}^{\prime \prime}(0)=u_{1}^{\prime \prime}(1)=0 \\
u_{2}(0)=u_{2}(1)=0
\end{array}\right.
$$

To establish the existence of positive solutions, we make the following assumptions:

$\left(\mathrm{H}_{1}\right) \mathrm{g}_{\mathrm{i}} \in \mathrm{C}\left[(0,1), \mathrm{R}^{+}\right], 0<\int_{0}^{1} \mathrm{~g}_{\mathrm{i}}(\mathrm{t}) \mathrm{dt}<+\infty, \mathrm{i}=1,2$;

$\left(\mathrm{H}_{2}\right) \mathrm{f}_{1}, \mathrm{f}_{2} \in \mathrm{C}\left[\mathrm{I} \times \mathrm{R}^{+} \times \mathrm{R}^{+}, \mathrm{R}^{+}\right]$.

We consider BVP $(3.1)$ in $C[0,1] \times C[0,1]$. Evidently, $(C[0,1],\|\cdot\|)$ is a Banach space with norm $\|\varphi\|=\max _{0 \leqslant \mathrm{t} \leqslant 1}|\varphi(\mathrm{t})|$. We construct cones $\mathrm{K}, \mathrm{P}$ by

$$
\begin{aligned}
& \mathrm{K}=\{\varphi \in \mathrm{C}[0,1] \mid \varphi(\mathrm{t}) \geqslant 0, \mathrm{t} \in[0,1]\}, \\
& \mathrm{P}=\{\varphi \in \mathrm{C}[0,1] \mid \varphi(\mathrm{t}) \geqslant \mathrm{t}(1-\mathrm{t})\|\varphi\|, \mathrm{t} \in[0,1]\} .
\end{aligned}
$$

Throughout the rest of paper, the partial ordering is always given by $\mathrm{K}$.

We start by some preliminaries and a lemma. Obviously, $\left(u_{1}, u_{2}\right) \in C^{4}(0,1) \times C^{2}[0,1]$ is a solution of $\operatorname{BVP}(3.1)$ if and only if $\left(u_{1}, u_{2}\right) \in C[0,1] \times C[0,1]$ is a solution of the following nonlinear integral system:

$$
\left\{\begin{array}{l}
u_{1}(t)=\int_{0}^{1} G_{1}(t, s) g_{1}(s) f_{1}\left(s, u_{1}(s), u_{2}(s)\right) d s, \\
u_{2}(t)=\int_{0}^{1} G_{2}(t, s) g_{2}(s) f_{2}\left(s, u_{1}(s), u_{2}(s)\right) d s,
\end{array}\right.
$$

where

$$
G_{1}(t, s)=\int_{0}^{1} G_{2}(t, \tau) G_{2}(\tau, s) d \tau, \quad G_{2}(t, s)= \begin{cases}t(1-s), & 0 \leqslant t \leqslant s \leqslant 1 \\ s(1-t), & 0 \leqslant s \leqslant t \leqslant 1\end{cases}
$$

It is an elementary fact that the function $G_{i}(t, s)$ has the following property:

$$
\frac{1}{30} t(1-t) s(1-s) \leqslant G_{i}(t, s)=G_{i}(s, t) \leqslant t(1-t)(\text { or } s(1-s)), \quad t, s \in[0,1]
$$


In order to solve (3.2), we consider the operator $\mathrm{N}: \mathrm{K} \times \mathrm{K} \rightarrow \mathrm{K} \times \mathrm{K}$ defined by

$$
\mathrm{Nu}=\left(\mathrm{N}_{1} \mathrm{u}, \mathrm{N}_{2} \mathrm{u}\right)
$$

where $N_{i}=T_{i} F_{i}, i=1,2$,

$$
\begin{aligned}
\left(T_{i} \varphi\right)(t) & =\int_{0}^{1} G_{i}(t, s) g_{i}(s) \varphi(s) d s, \quad \varphi \in K, \\
F_{i}\left(u_{1}, u_{2}\right)(t) & =f_{i}\left(t, u_{1}(t), u_{2}(t)\right), \quad u=\left(u_{1}, u_{2}\right) \in K \times K .
\end{aligned}
$$

Thus solving (3.2) is equivalent to finding fixed point of operator

$$
\mathrm{N}\left(\mathrm{u}_{1}, \mathrm{u}_{2}\right)=\left(\mathrm{T}_{1} \mathrm{~F}_{1} \mathrm{u}, \mathrm{T}_{2} \mathrm{~F}_{2} \mathrm{u}\right), \quad \mathrm{u} \in \mathrm{K} \times \mathrm{K} .
$$

Condition $\left(\mathrm{H}_{1}\right)$ and (3.3) imply that $\mathrm{T}_{i}: \mathrm{P} \rightarrow \mathrm{P}(\mathrm{i}=1,2)$ are two completely continuous linear $e^{-}$ positive operators with $e(t)=t(1-t)$. Thus by the famous Krein-Rutman theorem (see [14]), we assert that $r\left(T_{i}\right)>0$ and there exist $\varphi_{i}^{*} \in P(i=1,2)$ with $\left\|\varphi_{i}^{*}\right\|=1$ such that

$$
r\left(T_{i}\right) \varphi_{i}^{*}=T_{i} \varphi_{i}^{*}
$$

which can be rewritten in the form

$$
r\left(T_{i}\right) \varphi_{i}^{*}(t)=\int_{0}^{1} G_{i}(t, s) g_{i}(s) \varphi_{i}^{*}(s) d s
$$

for all $t \in[0,1]$. Define operators $B_{i}: K \rightarrow K$ by

$$
\left(\mathrm{B}_{i} \varphi\right)(\mathrm{t})=\int_{0}^{1} \varphi_{i}^{*}(s) g_{i}(s) \varphi(s) d s, \varphi \in K .
$$

Since $\varphi_{i}^{*} \in \mathrm{P}$ is the positive eigenfunction of $\mathrm{T}_{i}$, it follows from (3.3) that

$$
\varphi_{i}^{*}(t) \geqslant \frac{1}{30 r\left(T_{i}\right)} t(1-t) \int_{0}^{1} s(1-s) g_{i}(s) \varphi_{i}^{*}(s) d s,
$$

and

$$
\varphi^{*}(\mathrm{t}) \leqslant \frac{1}{\mathrm{r}\left(\mathrm{T}_{i}\right)} \int_{0}^{1} s(1-s) g_{i}(s) \varphi_{i}^{*}(s) d s,
$$

therefore $\int_{0}^{1} s(1-s) g_{i}(s) \varphi_{i}^{*}(s) d s>0$. Set $\delta_{i}=\frac{1}{30 r\left(T_{i}\right)} \int_{0}^{1} s(1-s) g_{i}(s) \varphi_{i}^{*}(s) d s>0$. By the help of (3.4), we have

$$
\delta_{i} G_{i}(t, s) \leqslant \varphi_{i}^{*}(s) .
$$

Lemma 3.1. Let

$$
P_{i}=\left\{\varphi \in K \mid\left(B_{i} \varphi\right)(t) \geqslant r\left(T_{i}\right) \delta_{i}\|\varphi\|\right\}
$$

Then $\mathrm{T}_{\mathrm{i}}(\mathrm{K}) \subset \mathrm{P}_{\mathrm{i}}$.

Proof. It follows from (3.5) that for every $\varphi \in \mathrm{K}$

$$
\begin{aligned}
\left(\mathrm{B}_{i} \mathrm{~T}_{i} \varphi\right)(\mathrm{t}) & =\int_{0}^{1} \varphi_{i}^{*}(s) g_{i}(s)\left(T_{i} \varphi\right)(s) \mathrm{d} s \\
& =\int_{0}^{1} \varphi_{i}^{*}(s) g_{i}(s) \int_{0}^{1} G_{i}(s, \tau) g_{i}(\tau) \varphi(\tau) d \tau d s
\end{aligned}
$$




$$
\begin{aligned}
& =\int_{0}^{1} g_{i}(\tau) \varphi(\tau) \int_{0}^{1} G_{i}(\tau, s) g_{i}(s) \varphi_{i}^{*}(s) d s d \tau \\
& =r\left(T_{i}\right) \int_{0}^{1} \varphi_{i}^{*}(\tau) g_{i}(\tau) \varphi(\tau) d \tau \\
& \geqslant r\left(T_{i}\right) \delta_{i} \int_{0}^{1} G_{i}(t, \tau) g_{i}(\tau) \varphi(\tau) d \tau=r\left(T_{i}\right) \delta_{i}\left(T_{i} \varphi\right)(t) .
\end{aligned}
$$

Then $\left(B_{i} T_{i} \varphi\right)(t) \geqslant r\left(T_{i}\right) \delta_{i}\left\|T_{i} \varphi\right\|$, i.e., $T_{i}(K) \subset P_{i}$.

Theorem 3.2. Suppose that the conditions $\left(\mathrm{H}_{1}\right)-\left(\mathrm{H}_{2}\right)$ are satisfied and that for each $i \in\{1,2\}$ one of the following conditions is satisfied:

$\left(\mathrm{H}_{3}\right)$ There exist $\mathrm{k}_{\mathrm{i} 1} \in(1,+\infty), \mathrm{k}_{\mathrm{i} 2} \in[0,1), \mathrm{r}_{\mathrm{i}}, \mathrm{M}_{\mathrm{i}} \in(0,+\infty)$ such that

$$
\begin{aligned}
& f_{i}\left(t, u_{1}, u_{2}\right) \geqslant \frac{k_{i 1}}{r\left(T_{i}\right)} u_{i}, \quad u_{i} \leqslant r_{i}, \\
& f_{i}\left(t, u_{1}, u_{2}\right) \leqslant \frac{k_{i 2}}{r\left(T_{i}\right)} u_{i}+M_{i} ;
\end{aligned}
$$

$\left(\mathrm{H}_{4}\right)$ There exist $\mathrm{k}_{\mathrm{i} 1} \in[0,1), \mathrm{k}_{\mathrm{i} 2} \in(1,+\infty), \mathrm{r}_{\mathrm{i}}, \mathrm{M}_{\mathrm{i}} \in(0,+\infty)$ such that

$$
\begin{aligned}
& f_{i}\left(t, u_{1}, u_{2}\right) \leqslant \frac{k_{i 1}}{r\left(T_{i}\right)} u_{i}, \quad u_{i} \leqslant r_{i}, \\
& f_{i}\left(t, u_{1}, u_{2}\right) \geqslant \frac{k_{i 2}}{r\left(T_{i}\right)} u_{i}-M_{i} .
\end{aligned}
$$

Then (3.1) has a positive solution.

Proof. Notice that

$$
\begin{aligned}
\left(B_{i} N_{i} u\right)(t) & =\int_{0}^{1} \varphi_{i}^{*}(s) g_{i}(s)\left(T_{i} F_{i} u\right)(s) d s \\
& =\int_{0}^{1} \varphi_{i}^{*}(s) g_{i}(s) \int_{0}^{1} G_{i}(s, \tau) g_{i}(\tau) F_{i} u(\tau) d \tau d s \\
& =\int_{0}^{1} g_{i}(\tau) F_{i} u(\tau) \int_{0}^{1} G_{i}(\tau, s) g_{i}(s) \varphi_{i}^{*}(s) d s d \tau \\
& =r\left(T_{i}\right) \int_{0}^{1} \varphi_{i}^{*}(\tau) g_{i}(\tau) F_{i} u(\tau) d \tau \\
& =r\left(T_{i}\right)\left(B_{i} F_{i} u\right)(t) .
\end{aligned}
$$

Then Theorem 3.2 follows from Theorem 2.5 and Lemma 3.1 with $e_{i}=1$ and $B_{i 1}=B_{i 2}=B_{i}$.

\section{Acknowledgment}

The research was supported by the National Natural Science Foundation of China under grant (11371221, 11571207), and the Tai'shan Scholar Engineering Construction Fund of Shandong Province of China. 


\section{References}

[1] R. P. Agarwal, N. Hussain, M.-A. Taoudi, Fixed point theorems in ordered Banach spaces and applications to nonlinear integral equations, Abstr. Appl. Anal., 2012 (2012), 15 pages. 1

[2] S. Budişan, R. Precup, Positive solutions of functional-dierential systems via the vector version of Krasnoselskiis fixed point theorem in cones, Carpathian J. Math., 27 (2011), 165-172.

[3] X. Cheng, Existence of positive solutions for a class of second-order ordinary differential systems, Nonlinear Anal., 69 (2008), 3042-3049.

[4] X. Cheng, H. Lü, Multiplicity of positive solutions for a $\left(\mathrm{p}_{1}, \mathrm{p}_{2}\right)$-Laplacian system and its applications, Nonlinear Anal. Real World Appl., 13 (2012), 2375-2390. 1, 2.2

[5] X. Cheng, Z. Zhang, Existence of positive solutions to systems of nonlinear integral or differential equations, Topol. Methods Nonlinear Anal., 34 (2009), 267-277.

[6] X. Cheng, C. Zhong, Existence of positive solutions for a second-order ordinary differential system, J. Math. Anal. Appl., 312 (2005), 14-23.

[7] Y. Cui, L. Liu, X. Zhang, Uniqueness and Existence of Positive Solutions for Singular Differential Systems with Coupled Integral Boundary Value Problems, Abstr. Appl. Anal., 2013 (2013), 9 pages. 1

[8] K. Deimling, Nonlinear Functional Analysis, Springer-Verlag, Berlin, (1985). 1, 2, 2.1

[9] S. Djebali, T. Moussaoui, R. Precup, Fourth-order p-Laplacian nonlinear systems via the vector version of Krasnoselskii's fixed point theorem, Mediterr. J. Math., 6 (2009), 447-460. 1

[10] D. J. Guo, V. Lakshmikantham, Nonlinear Problems in Abstract Cones, Academic Press, Boston, (1988). 1, $2,2.1$

[11] N. Hussain, A. R. Khan, R. P. Agarwal, Krasnosel'skii and Ky Fan type fixed point theorems in ordered Banach spaces, J. Nonlinear Convex Anal., 11 (2010), 475-489. 1

[12] M. A. Krasnosel'skiǔ, Positive Solutions of Operator Equations, P. Noordhoff Ltd., Groningen, (1964). 2.4

[13] M. A. Krasnosel'ski1̌, Topological Methods in the Theory of Nonlinear Integral Equations, The Macmillan, New York, (1964). 2.4

[14] M. G. Kreĭn, M. A. Rutman, Linear operators leaving invariant a cone in a Banach space, Amer. Math. Soc. Transl., 10, (1962), 199-325. 3

[15] K. Q. Lan, W. Lin, Multiple positive solutions of systems of Hammerstein integral equations with applications to fractional differential equations, J. Lond. Math. Soc., 83 (2011), 449-469. 1

[16] R. Ma, Multiple nonnegative solutions of second-order systems of boundary value problems, Nonlinear Anal., 42 (2000), 1003-1010. 1

[17] M. Meehan, D. O'Regan, Positive $\mathrm{L}^{\mathrm{p}}$ solutions of Hammerstein integral equations, Arch. Math., 76 (2001), $366-376$.

[18] R. Precup, A vector version of Krasnoselskiis fixed point theorem in cones and positive periodic solutions of nonlinear systems, J. Fixed Point Theory Appl., 2 (2007), 141-151. 1

[19] R. Precup, Moser-Harnack inequality, Krasnoselskii type fixed point theorems in cones and elliptic problems, Topol. Methods Nonlinear Anal., 40 (2012), 301-313.

[20] J. R. L. Webb, Nonlocal conjugate type boundary value problems of higher order, Nonlinear Anal., 71 (2009), $1933-1940$.

[21] J. R. L. Webb, Solutions of nonlinear equations in cones and positive linear Operators, J. Lond. Math. Soc., 82 (2010), 420-436. 2.4

[22] Z. Yang, Positive solutions for a system of p-Laplacian boundary value problems, Comput. Math. Appl., 62 (2011), 4429-4438.

[23] Z. Yang, Positive solutions for a system of nonlinear Hammerstein integral equations and applications, Appl. Math. Comput., 218 (2012), 11138-11150. 1

[24] Z. Yang, J. Sun, Positive solutions of boundary value problems for systems of nonlinear second order ordinary differential equations, Acta Math. Sinica, 47 (2004), 111-118.

[25] Y. Zou, L. Liu, Y. Cui, The Existence of Solutions for Four-Point Coupled Boundary Value Problems of Fractional Differential Equations at Resonance, Abstr. Appl. Anal., 2014 (2014), 8 pages. 1 